\title{
More than 6 Months of Teriparatide Treatment Was More Effective for Bone Union than Shorter Treatment Following Lumbar Posterolateral Fusion Surgery
}

Seiji Ohtori, Sumihisa Orita, Kazuyo Yamauchi, Yawara Eguchi, Nobuyasu Ochiai, Kazuki Kuniyoshi, Yasuchika Aoki, Junichi Nakamura, Masayuki Miyagi, Miyako Suzuki, Gou Kubota, Kazuhide Inage, Takeshi Sainoh, Jun Sato, Yasuhiro Shiga, Koki Abe, Kazuki Fujimoto, Hiroto Kanamoto, Gen Inoue, Kazuhisa Takahashi

Department of Orthopaedic Surgery, Graduate School of Medicine, Chiba University, Chiba, Japan

\section{Study Design: Retrospective case series.}

Purpose: To examine the most effective duration of teriparatide use for spinal fusion in women with postmenopausal osteoporosis. Overview of Literature: We reported that daily subcutaneous injection of teriparatide (parathyroid hormone) significantly improved bone union after instrumented lumbar posterolateral fusion (PLF) in women with postmenopausal osteoporosis when compared with oral administration of bisphosphonate. However, the most effective duration of teriparatide use for spinal fusion has not been explored.

Methods: Forty-five women with osteoporosis diagnosed with degenerative spondylolisthesis from one of the three treatment groups were evaluated based on: short-duration treatment (average, 5.5 months; $n=15$; daily subcutaneous injection of $20 \mu \mathrm{g}$ teriparatide), long-duration treatment (average, 13.0 months; $n=15$; daily subcutaneous injection of $20 \mu \mathrm{g}$ teriparatide), and bisphosphonate treatment (average, 13.0 months; $n=15$; weekly oral administration of $17.5 \mathrm{mg}$ risedronate). All patients underwent PLF with a local bone graft. Fusion rate and duration of bone union were evaluated 1.5 years after surgery.

Results: Bone union rate and average duration for bone union were $92 \%$ and 7.5 months in the long-duration treatment group, 80\% and 8.5 months in the short-duration treatment group, and $70 \%$ and 10.0 months in the bisphosphonate treatment group, respectively. Results of bone union rate and average duration for bone union in the teriparatide treatment groups were significantly superior to those in the bisphosphonate treatment group $(p<0.05)$; whereas, significantly superior results were observed in long-duration treatment group when compared with short-duration treatment group $(p<0.05)$.

Conclusions: Daily injection of teriparatide for bone union was more effective than oral administration of bisphosphonate. Furthermore, a longer period of teriparatide treatment for bone union was more effective than a shorter period of same treatment.

Keywords: Teriparatide; Osteoporosis; Surgery

Received Apr 14, 2015; Accepted Apr 14, 2015

Corresponding author: Seiji Ohtori

Department of Orthopaedic Surgery, Graduate School of Medicine, Chiba University,

1-8-1 Inohana, Chuo-ku, Chiba 260-8670, Japan

Tel: +81-43-226-2117, Fax: +81-43-226-2116, E-mail: sohtori@faculty.chiba-u.jp 


\section{Introduction}

Between 2004 and 2009 in the USA, national estimates for the annual number of discharged in-patients with a primary diagnosis of lumbar spinal stenosis and spondylolisthesis increased from 94,011 to 102,107 [1]. The rate of decompressions decreased from $58.5 \%$ to $49.2 \%$ in inpatient's population over this period of time, whereas the rate of fusions increased from $21.5 \%$ to $31.2 \%$ [1]. In the past, autologous iliac crest bone grafting, grafting with bone taken from the spine, ceramics, demineralized bone matrix, and bone morphogenetic proteins (BMPs) have been used for lumbar fusion [2]. The use of BMP and interbody devices has increased significantly from 2004 to 2009 and 33\% of fusions have used BMP in 2009 in the USA [1]. However, several authors have reported complications with BMPs, such as neck swelling, hematoma, and respiratory failure following cervical spine treatment; and ectopic bone formation, vertebral osteolysis, and postoperative radiculitis in the lumbar spine [3].

Parathyroid hormone (PTH) has potent anabolic effects on bone and is used for treatment of low bone mass in patients with osteoporosis [4,5]. Preclinical data also supports the use of PTH for lumbar spinal fusion [6-8]. In three animal posterolateral fusion (PLF) models, fusion rates using PTH were significantly faster than in an autograft only group [6-8]. The efficacy of teriparatide for fracture healing in the cervical spine has also been reported [9]. In the same report, three patients were observed to develop painful delayed unions of type III odontoid fractures despite external immobilization and all the three teriparatide-treated patients experienced both rapid clinical improvement and computed tomographic evidence of fracture union [9]. Daily subcutaneous injection of teriparatide for bone union in combination with local bone grafting after instrumented lumbar PLF in postmenopausal women with osteoporosis has been shown to be more effective than oral administration of bisphosphonate [10]. Furthermore, the efficacy of teriparatide treatment to reduce pedicle screw (PS) loosening after instrumented lumbar PLF in postmenopausal women with osteoporosis has been reported [11]. The incidence of PS loosening in the teriparatide group was significantly lower as compared with risedronate or the control group [11]. However, the most effective duration of teriparatide use for spinal fusion has not yet been explored.

The purpose of the current study was to examine the most effective duration of teriparatide use for spinal fusion in women with postmenopausal osteoporosis.

\section{Materials and Methods}

\section{Patients}

Between January 2009 and September 2012, we retrospectively evaluated 45 patients, 53-84 years old (mean \pm standard deviation, $71.0 \pm 7.0$ years), who had low back pain and leg pain with lumbar spinal stenosis and spondylolisthesis in Chiba University Hospital. Patients had low back and leg pain, continuing for at least 3 months. Patients were diagnosed with lumbar degenerative spondylolisthesis and spinal stenosis on X-ray and magnetic resonance imaging, myelography, and computed tomography (CT) after myelography. Patients, who had previously undergone spinal surgery, were excluded from the study. We also excluded those with spinal tumors, infections, or acute vertebral fractures of the thoracic and lumbar spine. Diagnosis of spondylolisthesis and inclusion criteria for fusion surgery were (1) more than 5\% slip of vertebra in a neutral position, or (2) more than $3 \mathrm{~mm}$ translation between flexion and extension positions on radiographic evaluation.

Informed consent was obtained from each of the participants. All of them were diagnosed as having osteoporosis based on Japanese criteria [12]. Bone mineral density (BMD) in the lumbar spine was examined before surgery. Details of the patients' backgrounds are shown in Table 1.

All patients underwent decompression and PLF surgery. One-level or two-level PLF was performed using PSs and a local bone graft. Bilateral facet fusion was performed in all patients. Local bone consisted of lamina from the decompression site. We did not use other osteoconductive products for spinal fusion.

\section{Administration of teriparatide or risedronate}

Some patients were treated with bisphosphonate for osteoporosis (daily oral administration of $2.5 \mathrm{mg}$ risedronate; Eisai, Tokyo, Japan); however, most of the patients were not treated with antiosteoporotic treatment for osteoprosis. Subsequently, patients were administered teriparatide (Eli Lilly, Tokyo, Japan) for 3 months before surgery thus forming two teriparatide groups. Risedronate was administrated in risedronate group for 3 months before surgery 
Table 1. Demographic characteristics

\begin{tabular}{|c|c|c|c|c|}
\hline \multirow[b]{2}{*}{ Variable } & \multirow[b]{2}{*}{ Risedronate } & \multicolumn{2}{|c|}{ Teriparatide } & \multirow[b]{2}{*}{$p$-value } \\
\hline & & $\begin{array}{l}\text { Short-duration } \\
\text { treatment group }\end{array}$ & $\begin{array}{l}\text { Long-duration } \\
\text { treatment group }\end{array}$ & \\
\hline No. of patients & 15 & 15 & 15 & - \\
\hline Sex (female), n (\%) & $15(100)$ & $15(100)$ & $15(100)$ & - \\
\hline Age (yr) & $71 \pm 6.0(58-84)$ & $69 \pm 7.1(53-79)$ & $72 \pm 7.7(53-82)$ & 0.55 \\
\hline Symptom duration (mo) & $10.0(4-12)$ & $12.5(4-36)$ & $9.8(3-28)$ & 0.36 \\
\hline Follow-up after surgery (mo) & 15 & 15 & 15 & - \\
\hline BMD (young adult mean) (\%) & $66.8 \pm 8.0(55-70)$ & $63.0 \pm 7.8(59-69)$ & $65.6 \pm 6(62-70)$ & 0.33 \\
\hline Diabetes mellitus & 2 & 2 & 4 & 0.20 \\
\hline Smoker & - & - & - & - \\
\hline Antiosteoporotic treatment: bisphosphonate & 2 & 3 & 2 & \\
\hline \multicolumn{5}{|l|}{ Pain score before surgery } \\
\hline \multicolumn{5}{|l|}{ Low back pain } \\
\hline VAS & $4.5 \pm 2.4$ & $3.8 \pm 2.5$ & $3.6 \pm 2.1$ & 0.23 \\
\hline ODI & $42 \pm 12$ & $34 \pm 14$ & $32 \pm 18$ & 0.33 \\
\hline \multicolumn{5}{|l|}{ Leg pain } \\
\hline VAS & $9.0 \pm 3.6$ & $7.9 \pm 2.0$ & $8.2 \pm 2.3$ & 0.32 \\
\hline
\end{tabular}

Values are presented as mean \pm standard deviation (range) unless otherwise indicated.

A $p<0.05$ was considered statistically significant.

$\mathrm{BMD}$, bone mineral density; VAS, visual analogue scale; ODI, Oswestry disability index.

(weekly oral administration of $17.5 \mathrm{mg}$ risedronate). After surgery, patients were administered either teriparatide or bisphosphonate.

Patients (group, $\mathrm{n}=15$ ) were retrospectively classified into a short-duration treatment group (average, 5.5 months; range, 4-6 months) with daily subcutaneous injections of $20 \mathrm{mg}$ of teriparatide; a long-duration treatment group (average, 13.0 months; range, 8-18 months) with daily subcutaneous injections of $20 \mathrm{mg}$ of teriparatide; and a bisphosphonate treatment group (average, 13.0 months; range, 11-18 months) with weekly oral administration of $17.5 \mathrm{mg}$ risedronate. Less than 6 months of teriparatide administration was defined as short-duration treatment, and more than 6 months of use was defined as long-duration treatment. After treatment with teriparatide, the patients were treated with $17.5 \mathrm{mg}$ of risedronate again until final follow-up 15 months after surgery.

\section{Radiographic and clinical evaluation}

Radiography was used to evaluate bone union. Profile views of X-ray images in flexion and extension before and $3,6,9,12$, and 15 months after surgery were evaluated. We defined bone union as less than $1.5^{\circ}$ instability between the flexion and extension positions. CT was performed to evaluate bone union 6 and 12 months after surgery. In the present scenario, we defined bone union as bone formation bridging the transverse processes between adjacent vertebrae on each side separately. We classified bone union or failure to fuse at each surgical level. We also evaluated the extent of fusion of the facet joints. Average periods for bone union were evaluated using profile views from X-ray images with the patient in flexion and extension position. If the patient underwent two level fusions and showed only one level fusion, we considered this pattern as "fused" in evaluating duration of treatment for bone union. Evaluation of bone union was blinded and performed by three surgeons independently. If at least two of the observers concurred, we considered occurrence of fusion and defined completion of bone union.

We evaluated the change in low back and leg pain before surgery. To evaluate pain, the visual analogue scale 
(VAS) score (0, no pain; 10, worst pain) for low back pain and leg pain, and the Oswestry disability index (ODI) for low back pain were recorded and compared.

\section{Adverse events}

All adverse events were reported together with an assessment of their severity (mild, moderate, and severe) and the investigator's opinion of their relationship to the treatment with each drug (none, unlikely, possible, or probable).

\section{Statistical analyses}

A Kruskal-Wallis test was used to compare pain scale data between the three groups. A one-way analysis of variance with post-hoc comparisons was used for age, symptom duration, and follow-up data, and a Fisher test was used for dichotomous or categorical variables. SPSS ver. 16.0 (SPSS Inc., Chicago, IL, USA) was used for statistical analysis. The Bonferroni correction was used for post-hoc pairwise comparisons between groups. A $p<0.05$ was considered statistically significant.

\section{Results}

Table 1 shows demographic characteristics of the patients before surgery. There was no significant difference among the three groups with respect to patients' background, low back pain score evaluated by VAS and ODI, or leg pain score evaluated by VAS $(p>0.05)$. Moreover, there was no significant difference among the three groups in terms of number of patients with diabetes mellitus or the one's, who smoked $(p>0.05)$. No significant difference in BMD was seen among the three groups ( $p>0.05$ ). In addition, 3 months prior to surgery, no significant difference in use of antiosteoporotic treatments among the three groups was noted $(p>0.05)$.

Ten patients in the risedronate group had one fusion

Table 2. Number of fusion levels and evaluation of bone union

\begin{tabular}{|c|c|c|c|c|c|c|}
\hline \multirow[b]{2}{*}{ Variable } & \multirow{2}{*}{$\begin{array}{l}\text { Risedronate } \\
\text { (1) }\end{array}$} & \multicolumn{2}{|c|}{ Teriparatide } & \multicolumn{3}{|c|}{$p$-value ${ }^{\text {a) }}$} \\
\hline & & $\begin{array}{l}\text { Short-duration } \\
\text { treatment (2) }\end{array}$ & $\begin{array}{l}\text { Long-duration } \\
\text { treatment }(3)\end{array}$ & $(1) \&(2)$ & $(1) \&(3)$ & (2) \& (3) \\
\hline No. of patients & 15 & 15 & 15 & & & \\
\hline \multicolumn{7}{|l|}{ No. of fusion levels } \\
\hline 1 & 10 & 7 & 9 & & & \\
\hline 2 & 5 & 8 & 6 & & & \\
\hline \multicolumn{7}{|l|}{ Fusion across the transverse processes (CT) } \\
\hline No. of segments at $12 \mathrm{mo}(\%)$ & $28(70)$ & $36(78)$ & $36(86)$ & 0.04 & 0.02 & 0.045 \\
\hline No fusion (\%) & $12(30)$ & $10(22)$ & $6(14)$ & 0.045 & 0.01 & 0.03 \\
\hline Total no. of evaluated segments & 40 & 46 & 42 & & & \\
\hline \multicolumn{7}{|l|}{ Bone union of facet joints (CT) } \\
\hline No. of facet joints at $12 \mathrm{mo}(\%)$ & $20(50)$ & $28(61)$ & $34(81)$ & - & 0.033 & 0.033 \\
\hline Total no. of evaluated facet segments & 40 & 46 & 42 & & & \\
\hline \multicolumn{7}{|l|}{$\begin{array}{l}\text { Bone union by dynamic } X \text {-ray } \\
\text { (no. of segments at (\%) at } 15 \mathrm{mo} \text { ) }\end{array}$} \\
\hline Instability (-) & $14(70)$ & $19(83)$ & $19(90)$ & 0.035 & $<0.01$ & 0.044 \\
\hline Instability (+) & $6(30)$ & $4(17)$ & $2(10)$ & 0.035 & $<0.01$ & 0.02 \\
\hline Total no. of evaluated facet segments & 20 & 23 & 21 & & & \\
\hline $\begin{array}{l}\text { Average period to bone union by dynamic } \\
\text { X-ray (mo) }\end{array}$ & $11.40 \pm 3.60(6-15)$ & $9.27 \pm 3.30(6-15)$ & $7.71 \pm 2.50(3-12)$ & 0.025 & 0.01 & 0.03 \\
\hline
\end{tabular}

Values are presented as number (\%) or mean \pm SD (range).

CT, computed tomography; SD, standard deviation.

${ }^{\text {a) }} \mathrm{A} p<0.05$ was considered statistically significant. The $p$-values represent the differences between groups. 
and five patients had two levels fused; seven patients in the short-duration teriparatide treatment group had one fusion and eight patients in this group had two levels fused; and nine patients in the long-duration treatment group had one fusion, whereas six patients had two fusions. There was no significant difference among the groups in total number of fusion levels ( $p>0.05)$ (Table 2). PLF and facet fusion were performed bilaterally, so the total number of evaluated segments and facet joints by CT were 40,46 , and 42 , respectively.

At 12 months follow-up by CT, 70\% of segments exhibited fusion across the transverse processes in the risedronate group; $78 \%$ of segments exhibited fusion in the short-duration teriparatide treatment group; and $86 \%$ of segments exhibited fusion in the long-duration treatment group. The rates of bone fusion in the teriparatide groups were significantly greater than the risedronate group $(p<0.05)$ (Table 2). The rate of bone fusion in the longduration teriparatide treatment group was significantly greater when compared to short-duration treatment group $(p<0.05)$ (Table 2$)$.

At 12 months follow-up by CT, $50 \%$ of facet joints had achieved bone union in the risedronate group; $61 \%$ had achieved union in the short-duration teriparatide treatment group; and $81 \%$ had achieved union in the longduration treatment group. The rates of bone fusion in the long-duration teriparatide treatment group were significantly greater than the risedronate and short-duration treatment groups $(p<0.05)$ (Table 2, Fig. 1).

Average periods of bone union in the teriparatide treat-
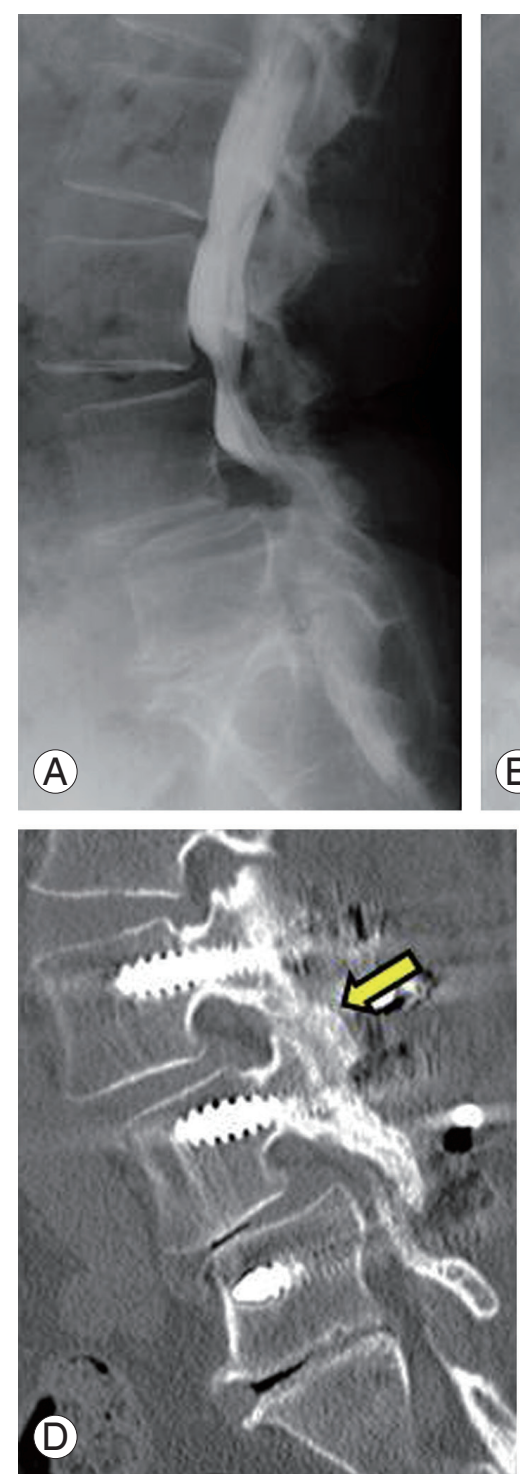
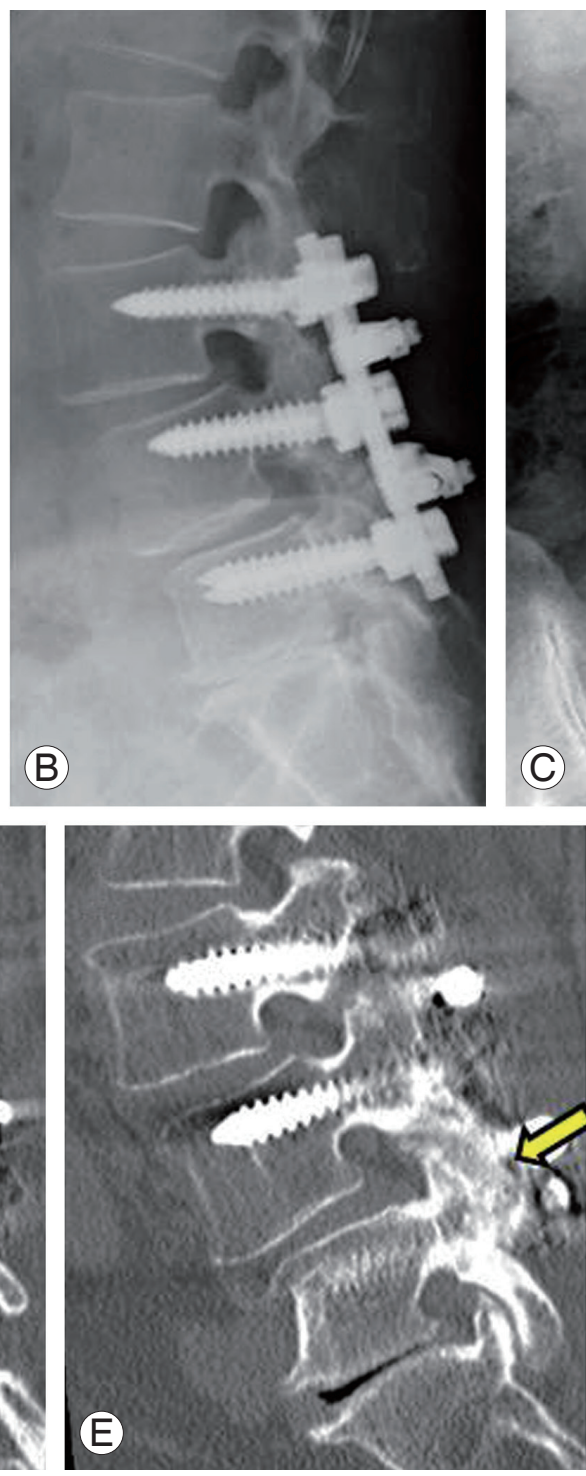

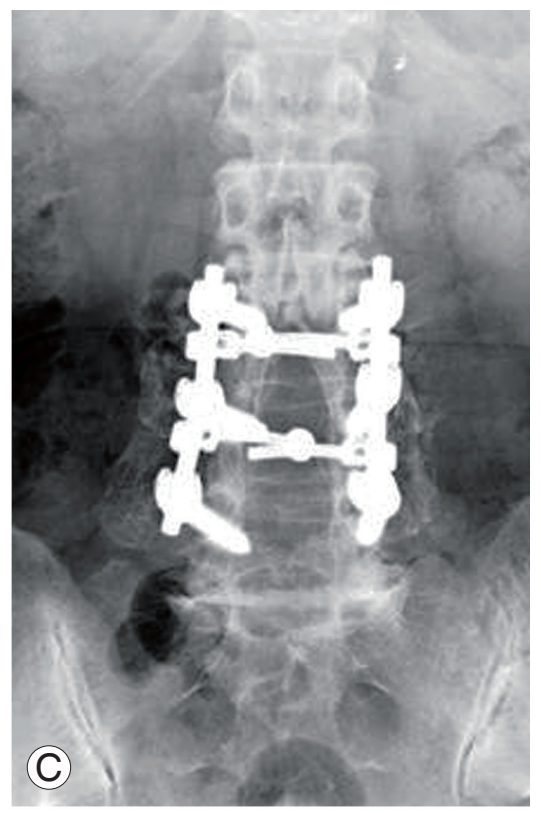

Fig. 1. Patient in teriparatide longduration treatment group. (A) Myelogram before surgery. (B) The patient showed $\mathrm{L} 3$ and $\mathrm{L} 4$ degenerated spondylolisthesis and spinal stenosis. (C) X-ray at 15 months after surgery. Bilateral bone formation bridging the transverse processes between adjacent vertebrae was seen in this patient. (D, E) Computed tomography at 12 months after surgery. Facet fusions (arrows) were observed in this patient. 
ment groups evaluated by X-ray profile views in flexion and extension at $3,6,9,12$, and 15 months after surgery were significantly shorter than the risedronate group $(p<0.05)$ (Table 2). Furthermore, the average period for bone union in the long-duration teriparatide treatment group was significantly shorter when compared to shortduration treatment group $(p<0.05)$ (Table 2).

There were no adverse events in any group.

\section{Discussion}

In the current study, daily subcutaneous injection of teriparatide for bone union was found to be more effective than oral administration of bisphosphonate. Furthermore, a longer period of teriparatide treatment was observed to be more effective in terms of achieving bone union than a short period of teriparatide treatment.

Two clinical trials of bone fracture healing in the distal radius or in the pelvis using teriparatide have been reported in 2010 and 2011, respectively [13,14]. Postmenopausal women, who had sustained a dorsally angulated distal radial fracture in need of closed reduction without surgery were randomly assigned to 8 weeks of once-daily injections of placebo or teriparatide [13]. The investigators concluded that healing time was shorter in the teriparatide-treated group [12]. In the latter study, patients aged 65 with osteoporosis had pelvic fractures. In this patient cohort, 21 patients received a once-daily injection of 100 $\mu \mathrm{g}$ PTH (1-84) starting within 2 days after admission to the hospital, and 44 patients served as the control group [14]. PTH 1-84 was observed to accelerate fracture-healing in pelvic fractures and improved the functional outcome [14]. For spine fusion, it has been shown that daily subcutaneous injection of teriparatide in combination with local bone grafting after instrumented lumbar PLF in postmenopausal women with osteoporosis was more effective than oral administration of bisphosphonate [10]. Teriparatide was administered for a total of 10 months (2 months before and 8 months after surgery). However, the most effective duration of teriparatide use for spinal fusion was not explored in that study.

It has been reported that subcutaneous administration of $20 \mu \mathrm{g} /$ day of teriparatide over a treatment period of 11-21 months improved BMD and reduced the risk of fractures in women with postmenopausal osteoporosis [15]. Furthermore, the beneficial effects of teriparatide on vertebral fracture prevention and BMD appear to persist following treatment cessation [15]. BMD increased by $9 \%$ at the lumbar spine with 12 months of teriparatide treatment, and $14 \%$ with 24 months of teriparatide treatment in Japanese subjects [16]. Evaluation of vertebral body architecture has been evaluated in another study, in which subjects received $20 \mu \mathrm{g} /$ day teriparatide for 18 months with quantitative computed tomography scans being performed at 0,6 , and 18 months [17]. At 18 months, high-resolution quantitative computed tomography showed significant improvement in trabecular and cortical variables during treatment with teriparatide, and the effects were correlated with length of treatment [17]. Fracture incidence, back pain, and quality of life measures were evaluated in a Greek population of postmenopausal osteoporotic women treated with teriparatide for 6,12 , or 18 months [18]. Significant improvements in quality of life and back pain together with low fracture rates were recorded, and the effects were correlated with length of treatment [18]. In the current study, the rate of bone union and average duration of treatment to bone union in patients in the long-duration teriparatide treatment group were significantly superior when compared to the short-duration treatment group. It is proposed that longer treatment durations may improve bony union rates after surgery, and accelerate healing.

The current study has some limitations. First, it is a retrospective study based on a small sample, and the number of patients was restricted. Second, BMD in the lumbar spine was examined before surgery, but we did not examine BMD after surgery. It would have been better to examine BMD after surgery; however, due to instrumentation problems we could not get accurate BMD data. Third, we did not evaluate pain scores at final follow-up, although some authors have reported a discrepancy between bone union and clinical results [19,20]. Fischgrund et al. [19] reported that successful arthrodesis did not correlate with the patients' outcome. Herkowitz and Kurz [20] reported presence of pseudoarthrosis of the fusion mass in $36 \%$ of patients and the clinical results were excellent for these patients. Indeed, although there was a significant difference in fusion rate between teriparatide-treatment and bisphosphonate-treatment in our recent study, no significant difference in the pain score between the two groups at final follow-up was noted $[10,11]$. Fourth, we used bisphosphonates after treatment with teriparatide for ethical reasons. Fifth, this study was a retrospective study and the number of patients was small. There was no 
significant difference among the three groups with respect to patients' characteristics, such as BMD, pain score, or on information if they received treatment for osteoporosis before the initiation of study. However, we cannot deny the possibility of selection bias in the current study. Finally, we were not able to evaluate an unmedicated control group. Further study is required to clarify the aforementioned points.

\section{Conclusions}

For instrumented lumbar PLF in postmenopausal women with osteoporosis, more than 6 months of daily teriparatide injection accelerated bone union when compared with less than 6 months of teriparatide treatment or treatment with bisphosphonate.

\section{Conflict of Interest}

No potential conflict of interest relevant to this article was reported.

\section{References}

1. Bae HW, Rajaee SS, Kanim LE. Nationwide trends in the surgical management of lumbar spinal stenosis. Spine (Phila Pa 1976) 2013;38:916-26.

2. Miyazaki M, Tsumura H, Wang JC, Alanay A. An update on bone substitutes for spinal fusion. Eur Spine J 2009;18:783-99.

3. McGovern SC, Fong W, Wang JC. Can bone morphogenetic protein binding peptide increase efficiency of bone formation? Spine (Phila Pa 1976) 2010;35:16559.

4. Dempster DW, Cosman F, Kurland ES, et al. Effects of daily treatment with parathyroid hormone on bone microarchitecture and turnover in patients with osteoporosis: a paired biopsy study. J Bone Miner Res 2001;16:1846-53.

5. Neer RM, Arnaud CD, Zanchetta JR, et al. Effect of parathyroid hormone (1-34) on fractures and bone mineral density in postmenopausal women with osteoporosis. N Engl J Med 2001;344:1434-41.

6. Lehman RA Jr, Dmitriev AE, Cardoso MJ, et al. Effect of teriparatide $[\operatorname{rhPTH}(1,34)]$ and calcitonin on intertransverse process fusion in a rabbit model. Spine (Phila Pa 1976) 2010;35:146-52.
7. O’Loughlin PF, Cunningham ME, Bukata SV, et al. Parathyroid hormone (1-34) augments spinal fusion, fusion mass volume, and fusion mass quality in a rabbit spinal fusion model. Spine (Phila Pa 1976) 2009;34:121-30.

8. Abe Y, Takahata M, Ito M, Irie K, Abumi K, Minami A. Enhancement of graft bone healing by intermittent administration of human parathyroid hormone (1$34)$ in a rat spinal arthrodesis model. Bone 2007;41: 775-85.

9. Rubery PT, Bukata SV. Teriparatide may accelerate healing in delayed unions of type III odontoid fractures: a report of 3 cases. J Spinal Disord Tech 2010; 23:151-5.

10. Ohtori S, Inoue G, Orita S, et al. Teriparatide accelerates lumbar posterolateral fusion in women with postmenopausal osteoporosis: prospective study. Spine (Phila Pa 1976) 2012;37:E1464-8.

11. Ohtori S, Inoue G, Orita S, et al. Comparison of teriparatide and bisphosphonate treatment to reduce pedicle screw loosening after lumbar spinal fusion surgery in postmenopausal women with osteoporosis from a bone quality perspective. Spine (Phila $\mathrm{Pa}$ 1976) 2013;38:E487-92.

12. Soen S, Fukunaga M, Sugimoto T, et al. Diagnostic criteria for primary osteoporosis: year 2012 revision. J Bone Miner Metab 2013;31:247-57.

13. Aspenberg P, Genant HK, Johansson T, et al. Teriparatide for acceleration of fracture repair in humans: a prospective, randomized, double-blind study of 102 postmenopausal women with distal radial fractures. J Bone Miner Res 2010;25:404-14.

14. Peichl P, Holzer LA, Maier R, Holzer G. Parathyroid hormone 1-84 accelerates fracture-healing in pubic bones of elderly osteoporotic women. J Bone Joint Surg Am 2011;93:1583-7.

15. Blick SK, Dhillon S, Keam SJ. Teriparatide: a review of its use in osteoporosis. Drugs 2008;68:2709-37.

16. Miyauchi A, Matsumoto T, Sugimoto T, Tsujimoto M, Warner MR, Nakamura T. Effects of teriparatide on bone mineral density and bone turnover markers in Japanese subjects with osteoporosis at high risk of fracture in a 24-month clinical study: 12-month, randomized, placebo-controlled, double-blind and 12-month open-label phases. Bone 2010;47:493-502.

17. Gluer CC, Marin F, Ringe JD, et al. Comparative effects of teriparatide and risedronate in glucocorti- 
coid-induced osteoporosis in men: 18-month results of the EuroGIOPs trial. J Bone Miner Res 2013;28: 1355-68.

18. Aloumanis K, Karras D, Drossinos V, Korelis E, Polydorakis A. Fracture incidence, quality of life, and back pain during 18-months treatment with teriparatide in Greek postmenopausal women with osteoporosis: results from the European Forsteo Observational Study. J Osteoporos 2011;2011:510398.

19. Fischgrund JS, Mackay M, Herkowitz HN, Brower R, Montgomery DM, Kurz LT. 1997 Volvo Award winner in clinical studies: degenerative lumbar spondylolisthesis with spinal stenosis: a prospective, randomized study comparing decompressive laminectomy and arthrodesis with and without spinal instrumentation. Spine (Phila Pa 1976) 1997;22:280712.

20. Herkowitz HN, Kurz LT. Degenerative lumbar spondylolisthesis with spinal stenosis: a prospective study comparing decompression with decompression and intertransverse process arthrodesis. J Bone Joint Surg Am 1991;73:802-8. 